\title{
The risks and benefits of patients temporarily discontinuing medications in the event of an intercurrent illness: a systematic review protocol
}

\author{
Andrew Morden ${ }^{1,2^{*}}$, Jeremy Horwood ${ }^{1,2}$, Penny Whiting ${ }^{1,2}$, Jelena Savovic ${ }^{1,2}$, Laurie Tomlinson ${ }^{3}$, \\ Thomas Blakeman ${ }^{4,5}$, Charles Tomson ${ }^{6}$, Alison Richards ${ }^{1,2}$, Tracey Stone ${ }^{1,2}$ and Fergus Caskey ${ }^{2,7}$
}

\begin{abstract}
Background: Acute kidney injury (AKI) is common and often leads to significant morbidity and/or death. The development of AKI, or complications associated with it, may be due to use of certain medications in at-risk patients experiencing an intercurrent illness. Implicated drugs include diuretics, angiotensin-converting enzyme inhibitors/angiotensin receptor blockers/direct renin inhibitors, non-steroidal anti-inflammatory drugs (NSAIDs), metformin and sulfonylureas. Expert consensus opinion (and clinical guidelines) recommend considering discontinuation of diuretics, angiotensin-converting enzyme inhibitors/angiotensin receptor blockers/direct renin inhibitors, NSAIDs, metformin and sulfonylureas in the event of an intercurrent illness to prevent AKI onset or reduce severity or complications. However, the evidence base for these recommendations is very limited. This systematic review aims to address the available evidence for the temporary discontinuation of diuretics, ACE inhibitors, angiotensin receptor blockers, direct renin inhibitors, non-steroidal anti-inflammatories and metformin and sulfonylureas for those at risk of AKI or with newly diagnosed AKI.
\end{abstract}

Methods/Design: Randomised controlled trials; non-randomised trials; cohort studies; case-control studies; interrupted time series studies; and before-and-after studies featuring adults aged 18 and over in any setting currently taking diuretics, angiotensin-converting enzyme inhibitors/angiotensin receptor blockers/direct renin inhibitors, NSAIDs and metformin; experiencing an intercurrent illness; or undergoing a radiological/surgical procedure (planned or unplanned) will be searched for. Relevant trial registers and systematic review databases will be searched. Systematic reviews will be assessed for methodological quality using the ROBIS tool, trials will be assessed using the Cochrane risk of bias tool, and observational studies will be assessed using the ACROBAT-NRS tool. If sufficient studies assessing similar populations, study type, settings and outcomes are found, then a formal meta-analysis will be performed to estimate summary measures of effect. If not, a narrative synthesis will be adopted.

Discussion: This review will synthesise evidence for the efficacy of discontinuing diuretics, angiotensin-converting enzyme inhibitors/angiotensin receptor blockers/direct renin inhibitors, NSAIDs, metformin or sulfonylureas to prevent or delay onset of AKI or associated complications. Results will provide guidance on efficacy and safety of this strategy and potentially help to develop an intervention to test the best mechanism of guiding medication discontinuation in at-risk populations.

\section{Systematic review registration: PROSPERO CRD42015023210}

Keywords: Acute kidney injury, Medication discontinuation, Sick day rules, Diuretics, Angiotensin-converting enzyme inhibitors, Angiotensin receptor blockers, Direct renin inhibitors, NSAID, Metformin and sulfonylureas

\footnotetext{
* Correspondence: andrew.morden@bristol.ac.uk

${ }^{1}$ NIHR CLAHRC West, Bristol, UK

${ }^{2}$ School of Social and Community Medicine, University of Bristol, Bristol, UK

Full list of author information is available at the end of the article
}

(C) 2015 Morden et al. Open Access This article is distributed under the terms of the Creative Commons Attribution 4.0 International License (http://creativecommons.org/licenses/by/4.0/), which permits unrestricted use, distribution, and reproduction in any medium, provided you give appropriate credit to the original author(s) and the source, provide a link to the Creative Commons license, and indicate if changes were made. The Creative Commons Public Domain Dedication waiver (http://creativecommons.org/publicdomain/zero/1.0/) applies to the data made available in this article, unless otherwise stated. 


\section{Background}

Acute kidney injury (AKI) is a comparatively recent term describing a sudden reduction in renal excretory function. The term was introduced because of a lack of agreement about what compromised acute renal failure', with some definitions only including the requirement for renal replacement therapy. The causes of AKI can be divided into three groups: pre-renal, such as low blood pressure; intrinsic renal, commonly the result of prolonged sepsis or hypotension but also resulting from immune conditions affecting the kidney; and post-renal, caused by partial or complete obstruction to the flow of urine from the kidneys [1]. There are a number of key risk factors for AKI, including the following: existing chronic kidney disease (CKD), aged $>65$ years, cardiac failure, liver disease, diabetes mellitus, use of nephrotoxic drugs such as non-steroidal anti-inflammatories (NSAIDs) and aminoglycosides, hypotension (compared to baseline blood pressure) and sepsis, neurological or cognitive impairment or disability, or prior urological obstruction [1, 2]. An episode of AKI may be triggered by an intercurrent illness, such as diarrhoea and vomiting, influenza, other viruses, urinary tract infections, respiratory tract infections, skin infections and hypovolaemia from any cause in those most at risk $[1,3]$.

The pooled incidence rate of AKI globally is $21.6 \%$ in adults [4], and AKI is predicted to feature in $1-7 \%$ of hospital admissions $[1,3,5-7]$. The morbidity and mortality rates associated with an episode of AKI (in hospital and after discharge) are high [6, 8-10]. Coca et al.'s systematic review reports that the incidence of CKD and end-stage kidney disease (ESKD) after an AKI episode is 7.8 and 4.9/100 patient-years, respectively, and that AKI is associated with long-term mortality [10].

Pannu and colleagues review the potentially harmful biochemical effects of medications that are administered as part of the routine care of critical illnesses in hospital settings and conclude that many, such as angiotensinconverting enzyme (ACE) inhibitors and NSAIDS, could be detrimental to kidney function [9]. Medicationinduced episodes of kidney problems are increasingly prevalent and indeed doubled between 1999 and 2009 in the UK [11]. Commonly prescribed medications including diuretics, ACE inhibitors/angiotensin receptor blockers/direct renin inhibitors, NSAIDs, metformin and sulfonylureas have been associated with AKI (and related complications) in a variety of settings [12-30]. The incidence of AKI is higher amongst patients who have multiple comorbidities and who are taking combinations of drugs which may interact to enhance the physiological insult underlying AKI [31].

Expert consensus statements and clinical guidelines have recommended that clinicians temporarily withhold potentially harmful medications for high-risk patients when they develop an intercurrent illness to reduce risk of AKI [1-3]. Other interventions have gone further by suggesting that patients stop the medications when they become unwell [32]. However, there is surprisingly little evidence to support these recommendations. The National Institute for Health and Clinical Excellence (NICE) guidelines issued in 2013 found no studies which evaluate the efficacy of medication discontinuation during an intercurrent illness in terms of preventing onset or harm from AKI [2]. There is growing concern regarding the overall risks and benefits of the sick day rules approach [33]. Such advice could lead to an increase in complications from the conditions which the drugs are prescribed such as heart failure and hypertension. NSAIDs are recommended for pain control, especially for osteoarthritis pain in older adults [34]; cessation of NSAIDs may result in a marked reduction in mobility and make the difference between a person coping at home or not. In addition, it is suggested that continuing some of these drugs will have renal and cardiac benefits, even in the presence of AKI, at least in some patients. $\mathrm{ACE}$ inhibitors and angiotensin receptor blockers/direct renin inhibitors, for instance, preserve tubular blood flow and may prevent the tubular injury that characterises the transition from reversible pre-renal AKI to established, intrinsic renal AKI, which has a poorer prognosis [35].

Therefore, the overall objective of this review is to assess the available evidence for the temporary discontinuation of diuretics, ACE inhibitors, angiotensin receptor blockers, direct renin inhibitors, non-steroidal antiinflammatories, metformin and sulfonylureas in adults in primary and secondary care at risk of AKI or with newly diagnosed AKI as a result of an intercurrent illness or a radiological/surgical procedure (planned or unplanned). To address this objective, we will answer the following review questions:

1. What are the effects of temporary discontinuation of diuretics, angiotensin-converting enzyme inhibitors/ angiotensin receptor blockers/direct renin inhibitors, NSAIDs, metformin and sulfonylureas in those at risk of AKI?

2. What are the effects of the temporary discontinuation of diuretics, angiotensin-converting enzyme inhibitors/angiotensin receptor blockers/direct renin inhibitors, NSAIDs, metformin and sulfonylureas for the progression of AKI across AKI severity categories?

3. What are the adverse effects associated with stopping diuretics, angiotensin-converting enzyme inhibitors/angiotensin receptor blockers/direct renin inhibitors, NSAIDs, metformin and sulfonylureas? 
4. What is the effectiveness of interventions to encourage stopping of diuretics, angiotensinconverting enzyme inhibitors/angiotensin receptor blockers/direct renin inhibitors, NSAIDs, metformin and sulfonylureas?

\section{Methods/Design}

Systematic review methods will follow the principles outlined in the Centre for Reviews and Dissemination (CRD) guidance for undertaking reviews in health care [36] and the Cochrane Handbook [37]. The protocol is registered on the PROSPERO database (CRD42015023210). The review will be reported according to the PRISMA guidelines $[38,39]$. This protocol follows the PRISMA-P reporting guidelines [40].

\section{Eligibility criteria}

Studies that fulfil the following criteria will be eligible for inclusion:

\section{Types of studies}

The following types of studies will be included: randomised controlled trials, non-randomised trials, cohort studies, case-control studies, interrupted time series studies, before-and-after studies. If high-quality systematic reviews are identified that fulfil all inclusion criteria for the review, then these will be eligible for inclusion. We will include the systematic review if it includes all relevant studies for a single research question; if additional studies fulfil our inclusion criteria, then the original review will be used only as a source of relevant studies.

\section{Participants}

Only studies conducted in humans will be sought. Eligible participants will be adults aged 18 and over in any setting (e.g. community, GP surgery, hospital) currently taking diuretics, ACE inhibitors, angiotensin receptor blockers, direct renin inhibitors, non-steroidal anti-inflammatories, metformin or sulfonylureas; experiencing an intercurrent illness; or undergoing a radiological/surgical procedure (planned or unplanned).

\section{Interventions}

Temporary discontinuation of diuretics, angiotensinconverting-enzyme inhibitors/ angiotensin receptor blockers / direct renin inhibitors, NSAIDs, metformin and sulfonylureas as a result of a specific intervention or for any other reason. Eligible comparator interventions (if present) will be placebo, no treatment or usual care.

\section{Outcomes}

The review will be restricted to studies that report some measure of kidney function. We anticipate that other relevant outcomes will include all-cause mortality, cardiac events, diabetic events and pain-related outcomes (pain severity, use of GP services or admission to hospital relating to NSAID withdrawal).

\section{Search strategy}

Attempts will be made to identify relevant studies on temporarily discontinuing diuretics, angiotensin-converting enzyme inhibitors/angiotensin receptor blockers/direct renin inhibitors, NSAIDs, metformin and sulfonylureas. Search methods will meet best practice standards in systematic reviews [36, 37]. The search strategies will be developed specifically for each database and the keywords adapted according to the configuration of each database. Searches will not be limited by language, date or publication status (unpublished or published). Searches will be performed in multiple databases from inception to present, including Embase (OvidSP), Medline (OvidSP), Medline In-Process Citations \& Daily Update (OvidSP), PsycINFO (OvidSP), BIOSIS Citation Index (Web of Science), CINAHL (Cumulative Index to Nursing and Allied Health Literature) (EBSCO), Science Citation Index (SCI) (Web of Science) and Cochrane Central Register of Controlled Trials (CENTRAL) (Wiley).

Supplementary searches will be undertaken to identify grey literature, completed and ongoing trials, in resources such as NIH ClinicalTrials.gov (http://www.clinical trials.gov), metaRegister of Controlled Trials (http:// www.controlled-trials.com) and WHO International Clinical Trials Registry Platform (ICTRP) (http:// www.who.int/ictrp/en); relevant guidelines (i.e. NICE in the UK) regarding management of AKI; and references of published articles found in the search. An example of the search strategy is presented in Additional file 1.

Identified references will be downloaded into EndNote X7 software for further assessment and handling. Rigorous records are maintained as part of the searching process. Individual records within the EndNote reference libraries will be tagged with search information, such as searcher, date searched, database host, database searched, strategy name and iteration, theme, or search question. This will enable the information specialist to track the origin of each individual database record and its progress through the screening and review process. A review-specific access database will be used to manage screening and data extraction.

\section{Selection of studies}

Two reviewers will independently screen the titles and abstracts of all reports identified by searches, and any discrepancies will be discussed and resolved by consensus. Full copies of all studies deemed potentially relevant will be obtained, and the same two reviewers will 
independently assess these for inclusion; any disagreements will be resolved by consensus.

\section{Data extraction}

Data extraction will be carried out using standard data extraction forms designed specifically for this review. Data will be extracted by one reviewer, using a piloted, standard data extraction form, and checked by a second reviewer; any disagreements will be resolved by consensus. Data will be extracted on the following: participant characteristics, study design, inclusion and exclusion criteria, details of intervention (if applicable-potentially including an outline of the characteristics of the interventions in terms of the (1) format and content of any 'sick day rules' advice and (2) whether part of a wider package of care, i.e. in the context of other AKI/kidney health initiatives), details of outcomes assessed (primary and other outcome measures) and results. If, during the course of the review, outcome measures commonly reported in studies are found these will be included and documented [40].

\section{Quality assessment}

Systematic reviews will be assessed for risk of bias using the ROBIS tool [41]: this tool's aims include domains covering study eligibility criteria, identification and selection of studies, data collection and study appraisal, synthesis and findings, and interpretation. Trials will be assessed for methodological quality using the Cochrane risk of bias tool [37]. This includes items covering selection bias (random sequence generation and allocation concealment), performance bias (participant blinding), detection bias (blinding of outcome assessors), attrition bias (incomplete outcome data) and reporting bias (selective reporting). There is also an additional field for other sources of bias. We believe that all important concerns about bias are included in the other domains in the tool and so no further domains will be added. We will use the new ACROBAT-NRS tool to assess the risk of bias in observational studies [42]. It includes domains covering bias due to confounding, bias in the selection of participants into the study, bias due to departures from intended interventions, bias due to missing data, bias in taking measurements and bias in the selection of the reported result. For all tools, if at least one of the domains is rated as 'high', the study will be considered at high risk of bias; if all domains are judged as 'low', the trial will be considered at low risk of bias; otherwise, the trial will be considered at 'unclear' risk of bias. The risk of bias assessment will be conducted as part of the data extraction process.

\section{Data synthesis}

A narrative summary of the included studies will be presented. This will include a summary of the characteristics (e.g. study design, intervention (if applicable), population size, geographical location, settings (community, hospital or other healthcare settings), year, baseline population characteristics and outcome definition). If data are considered too heterogeneous to pool, or not reported in a format suitable for pooling (e.g. data reported as medians), then we will employ a narrative synthesis. This will involve the use of descriptive text and tables to summarise data in order to allow the reader to consider outcomes in the light of differences in study designs and potential sources of bias for each of the studies being reviewed. This involves organising the studies by (as appropriate) population, outcomes assessed, type of study and study settings; summarising the results of the studies, summarising the range and size of the associations these studies report; and describing the most important characteristics of the included studies. A detailed commentary on the major methodological problems or biases that affected the studies will also be included, together with a description of how this has affected the individual study results.

If sufficient studies assessing similar populations, study type, settings and outcomes are found, then a formal meta-analysis will be used to estimate summary measures of effect. For dichotomous data, we will calculate the relative risk (RR) for each study with the associated $95 \%$ confidence intervals. Continuous data will be analysed as the mean difference between groups and associated $95 \%$ confidence intervals. For multi-arm studies, we will analyse each intervention arm compared to the control group separately.

We anticipate that systematic differences between studies (heterogeneity) will be likely. Therefore, the random effects model will be used to calculate summary estimates. Heterogeneity will be investigated visually using forest plots and statistically using the $I^{2}$ and $Q$ statistics [43].

Heterogeneity will be formally investigated using metaregression analyses, where sufficient data are available. We anticipate that the effects of the following variables will be investigated: methodological quality of the primary studies, how the advice or decision to discontinue medications was administered and length of medication discontinuation, age, sex, and comorbidities. Other variables considered relevant on further examination of the literature or input from clinical experts may also be considered. The coefficient describing the effect of each variable on the outcome will be modelled, using a random effects model.

If appropriate, small-study effects (publication bias) will be assessed using a modified linear regression test for funnel plot asymmetry as recommended where there are sufficient numbers of trials (i.e. six trials) [44].

\section{Confidence in cumulative evidence}

We will consider using the GRADE guidelines [45] to help us evaluate the cumulative quality of the evidence 
reported in this review for risk of bias, publication bias, imprecision, inconsistency, indirectness and magnitude of effect.

\section{Discussion}

Diuretics, angiotensin-converting enzyme inhibitors, angiotensin receptor blockers, direct renin inhibitors, NSAIDs, metformin and sulfonylureas in at-risk patients experiencing an intercurrent illness are extremely commonly prescribed, and expert consensus (subsequently incorporated into clinical guidelines) recommends the discontinuation of these medications for individuals at risk of AKI during an intercurrent illness. The benefit from such advice is however unproven with scant evidence regarding the benefits and potential harms that discontinuation may confer. An up-to-date systematic review of the evidence on discontinuing medications is warranted. This review will provide evidence on the effectiveness and safety of discontinuing diuretics, angiotensin-converting enzyme inhibitors/angiotensin receptor blockers/direct renin inhibitors, NSAIDs, metformin and sulfonylureas in relation to AKI. We anticipate that the findings will aid healthcare practitioners and policymakers in developing recommendations regarding advice on temporary drug cessation in patients at risk of AKI. Furthermore, the findings will benefit researchers by highlighting future research directions through the identification of gaps in knowledge and shortcomings of the existing evidence base.

\section{Additional file}

Additional file 1: Search strategy Medline. (DOC 68 kb)

\section{Abbreviations}

ACE: angiotensin-converting enzyme; AKl: acute kidney injury; CKD: chronic kidney disease; CRD: Centre for Reviews and Dissemination; ESKD: end-stage kidney disease; NICE: National Institute for Health and Clinical Excellence; NSAIDS: non-steroidal anti-inflammatory drugs.

\section{Competing interests}

The authors declare that they have no competing interests.

\section{Authors' contributions}

$C T, T B$ and $F C$ conceived the idea for the review. AM drafted the written protocol with the support of FC, JS, PW and JH. All authors contributed to the development of the protocol. PW, JS and AR provided input on study methodology. FC served as a content expert in the field of AKI. JH served as the overall supervisor and provided input on study methodology. AM, TS and AR were involved in data acquisition. AM and TS undertook screening and data extraction. All authors contributed to data analysis and the interpretation of results. AM drafted the final manuscript intended for publication. All authors have given their approval for publication.

\section{Acknowledgements}

This research is supported by the National Institute for Health Research (NIHR) Collaboration for Leadership in Applied Health Research and Care West at University Hospitals Bristol NHS Foundation Trust. The views expressed are those of the author(s) and not necessarily those of the NHS, the NIHR or the Department of Health.

\section{Author details}

${ }^{1}$ NIHR CLAHRC West, Bristol, UK. ${ }^{2}$ School of Social and Community Medicine, University of Bristol, Bristol, UK. ${ }^{3}$ Department of Non-communicable Disease Epidemiology, London School of Hygiene and Tropical Medicine, London, UK. ${ }^{4}$ Centre for Primary Care, Institute of Population Health, The University of Manchester, Manchester, UK. ${ }^{5}$ NIHR CLAHRC Greater Manchester, Manchester, UK. ${ }^{6}$ Department of Renal Medicine, Freeman Hospital, Newcastle Upon Tyne Hospitals Foundation Trust, Tyne and Wear, UK. ${ }^{7}$ UK Renal Registry, Bristol, UK.

Received: 11 August 2015 Accepted: 15 October 2015

Published online: 24 October 2015

\section{References}

1. Prescott $A M$, Lewington $A, O^{\prime}$ Donoghue $D$. Acute kidney injury: top ten tips. Clin Med. 2012;12(4):328-32.

2. National Institute for Health and Clinical Excellence. Acute kidney injury: prevention, detection and management of acute kidney injury up to the point of renal replacement therapy. London: NICE; 2013.

3. Feehally J, Gilmore I, Barasi S, Bosomworth M, Christie B, Davies A, et al. RCPE UK consensus conference statement: management of acute kidney injury: the role of fluids, e-alerts and biomarkers. J R Coll Physicians Edinb. 2013;43(1):37-8.

4. Susantitaphong P, Cruz DN, Cerda J, Abulfaraj M, Alqahtani F, Koulouridis I, et al. World incidence of AKl: a meta-analysis. Clin J Am Soc Nephrol. 2013:8(9):1482-93.

5. National Confidential Enquiry into Patient Outcomes and Death. Adding insult to injury - a review of care of patients who died in hospital with a primary diagnosis of acute kidney injury (acute renal failure). London: National Confidential Enquiry into Patient Outcomes and Death; 2009.

6. Selby NM, Crowley L, Fluck RJ, Mclntyre CW, Monaghan J, Lawson N, et al. Use of electronic results reporting to diagnose and monitor AKI in hospitalized patients. Clin J Am Soc Nephrol. 2012;7(4):533-40.

7. Mehta RLPM, Soroko S, Savage BR, Himmelfarb J, Ikizler TA, Paganini EP, et al. Spectrum of acute renal failure in the intensive care unit: the PICARD experience. Kidney Int. 2004;66(4):1613-21.

8. Wang HE, Muntner P, Chertow GM, Warnock DG. Acute kidney injury and mortality in hospitalized patients. Am J Nephrol. 2012;35(4):349-55.

9. Pannu N, Nadim MK. An overview of drug-induced acute kidney injury. Crit Care Med. 2008;36(4 Suppl):S216-23.

10. Coca SG, Yusuf B, Shlipak MG, Garg AX, Parikh CR. Long-term risk of mortality and other adverse outcomes after acute kidney injury: a systematic review and meta-analysis. Am J Kidney Dis. 2009;53(6):961-73.

11. Wu TY, Jen MH, Bottle A, Molokhia M, Aylin P, Bell D, et al. Ten-year trends in hospital admissions for adverse drug reactions in England 1999-2009. J R Soc Med. 2010;103(6):239-50.

12. Tomlinson LA, Abel GA, Chaudhry AN, Tomson CR, Wilkinson IB, Roland MO, et al. ACE inhibitor and angiotensin receptor-II antagonist prescribing and hospital admissions with acute kidney injury: a longitudinal ecological study. PLoS One. 2013;8(11):e78465.

13. Saudan P, Alves C, De La Fuente V, Ponte B, Carballo S, Rutschmann O, et al. Risk factors for community-acquired acute kidney injury: a prospective observational study. Nephrol Dial Transplant. 2014;29:iii362.

14. Bickel M, Khaykin P, Stephan C, Schmidt K, Buettner M, Amann K, et al. Acute kidney injury caused by tenofovir disoproxil fumarate and diclofenac co-administration. HIV Med. 2013;14(10):633-8.

15. Eppenga WL, Lalmohamed A, Geerts AF, Derijks HJ, Wensing M, Egberts A et al. Risk of lactic acidosis or elevated lactate concentrations in metformin users with renal impairment: a population-based cohort study. Diabetes Care. 2014;37(8):2218-24.

16. Bouvy ML, Heerdink ER, Hoes AW, Leufkens HG. Effects of NSAIDs on the incidence of hospitalisations for renal dysfunction in users of ACE inhibitors. Drug Saf. 2003;26(13):983-9.

17. Calvo-Alen J, De Cos MA, Rodriguez-Valverde V, Escallada R, Florez J, Arias M. Subclinical renal toxicity in rheumatic patients receiving longterm treatment with nonsteroidal antiinflammatory drugs. J Rheumatol. 1994;21(9):1742-7.

18. Jang SM, Cerulli J, Grabe DW, Fox C, Vassalotti JA, Prokopienko AJ, et al. NSAID-avoidance education in community pharmacies for patients at high risk for acute kidney injury, upstate New York, 2011. Prev Chronic Dis. 2014;11:E220. 
19. Acharya M, Dunning J. Does the use of non-steroidal anti-inflammatory drugs after cardiac surgery increase the risk of renal failure? Interact Cardiovasc Thorac Surg. 2010;11(4):461-7.

20. Arora P, Rajagopalam S, Ranjan R, Kolli H, Singh M, Venuto R, et al. Preoperative use of angiotensin-converting enzyme inhibitors/angiotensin receptor blockers is associated with increased risk for acute kidney injury after cardiovascular surgery. Clin J Am Soc Nephrol. 2008;3(5):1266-73.

21. Bandeali SJ, Kayani WT, Lee W, Elayda M, Alam M, Huang HD, et al. Association between preoperative diuretic use and in-hospital outcomes after cardiac surgery. Cardiovasc Ther. 2013;31(5):291-7.

22. Barnes TC, Hernandez-Caballero C, Hurtado-Doce Al, Hall D. Risk factors for acute kidney injury post off pump coronary artery bypass graft (OP-CABG) surgery and its impact on shortterm mortality. Intensive Care Med. 2014:1:S256-7.

23. Curiel Balsera E, Olea Jimenez V, Munoz Bono J, Sanchez-Rodriguez AC, Dios-Torronteras F, Reina-Toral A, et al. Analysis of preoperative and operative predictors of acute kidney injury in cardiac surgery. A multicentre study. Intensive Care Med. 2013;39:S262.

24. Miceli A, Concistre G, Gilmanov D, Chiaramonti F, Murzi M, Varone E, et al. Renin-angiotensin antagonism systems and acute kidney injury after cardiac surgery. A meta analysis of over 20,000 patients. Heart Surg Forum. 2011;14:S107.

25. Saydy NT, Mazine A, Stevens L, Jeanmart H, Demers P, Page P, et al. Predictors of postoperative acute kidney injury in young adults undergoing cardiac surgery. Can J Cardiol. 2014;1:S239-40.

26. Andersen MB, Jensen MK, Palm H. Patient-safe pain management of hip fracture patients requires focus on the renal function. Int J Clin Pharm. 2015;37(1):273

27. Aveline C, Leroux A, Vautier P, Cognet F, Le Hetet H, Bonnet F. Risk factors for renal dysfunction after total hip arthroplasty. Ann Fr Anesth Reanim. 2009;28(9):728-34.

28. Kimmel LA, Janardan J, Liew SM, Walker RG. Incidence of acute kidney injury following total joint arthroplasty at a large tertiary teaching hospital: a retrospective review. Nephrology. 2014;19:46.

29. Nielson E, Hennrikus E, Lehman E, Mets B. Angiotensin axis blockade, hypotension, and acute kidney injury in elective major orthopedic surgery. J Hosp Med. 2014;9(5):283-8.

30. Dreischulte T, Morales DR, Bell S, Guthrie B. Combined use of nonsteroidal anti-inflammatory drugs with diuretics and/or renin-angiotensin system inhibitors in the community increases the risk of acute kidney injury. Kidney Int. 2015;88(2):396-403.

31. Lapi F, Azoulay L, Yin H, Nessim SJ, Suissa S. Concurrent use of diuretics, angiotensin converting enzyme inhibitors, and angiotensin receptor blockers with non-steroidal anti-inflammatory drugs and risk of acute kidney injury: nested case-control study. BMJ. 2013;346:e8525.

32. Medicine Sick Day Rules Card. [http://www.scottishpatientsafety programme.scot.nhs.uk/programmes/primary-care/medicine-sick-dayrules-card]

33. "Sick day rules" in patients at risk of acute kidney injury: an interim position statement from the Think Kidneys Board. [https://www.thinkkidneys.nhs.uk/ wp-content/uploads/2015/07/Think-Kidneys-Sick-Day-Rules-160715.pdf]

34. NICE. Ostearthiritis: National Clinical Guideline for Care and Management in Adults. London: Royal College of Physicians; 2014.

35. Perazella MA, Coca SG. Three feasible strategies to minimize kidney injury in 'incipient AKI'. Nat Rev Nephrol. 2013;9(8):484-90.

36. Systematic reviews: CRD's guidance for undertaking reviews in health care. [http://www.york.ac.uk/inst/crd/SysRev/ISSL!/WebHelp/SysRev3.htm]

37. Cochrane Handbook for Systematic Reviews of Interventions version 5.1.0. [http://handbook.cochrane.org/]

38. Liberati A, Altman DG, Tetzlaff J, Mulrow C, Gotzsche PC, loannidis JP, et al. The PRISMA statement for reporting systematic reviews and meta-analyses of studies that evaluate healthcare interventions: explanation and elaboration. BMJ. 2009;339:b2700.

39. Moher D, Liberati A, Tetzlaff J, Altman DG, Group P. Preferred reporting items for systematic reviews and meta-analyses: the PRISMA statement. BMJ. 2009;339:b2535.

40. Shamseer L, Moher D, Clarke M, Ghersi D, Liberati A, Petticrew M, et al. Preferred reporting items for systematic review and meta-analysis protocols (PRISMA-P) 2015: elaboration and explanation. BMJ. 2015;349:g7647.

41. Whiting P, Savovic J, Higgins JP, Caldwell DM, Reeves BC, Shea B, Davies P, Kleijnen J, Churchill R, group R. ROBIS: a new tool to assess risk of bias in systematic reviews was developed. J Clin Epidemiol. 2015 doi: 10.1016/j.jclinepi.2015.06.005.

42. A Cochrane risk of bias assessment tool: for non-randomized studies of interventions (ACROBAT- NRSI), version 1.0.0. [http://www.riskofbias.info]

43. Higgins JP, Thompson SG. Quantifying heterogeneity in a meta-analysis. Stat Med. 2002;21(11):1539-58.

44. Harbord RM, Egger M, Sterne JA. A modified test for small-study effects in meta-analyses of controlled trials with binary endpoints. Stat Med. 2006;25(20):3443-57.

45. Guyatt GH, Oxman AD, Schunemann HJ, Tugwell P, Knottnerus A. GRADE guidelines: a new series of articles in the Journal of Clinical Epidemiology. J Clin Epidemiol. 2011;64(4):380-2.

\section{Submit your next manuscript to BioMed Central and take full advantage of:}

- Convenient online submission

- Thorough peer review

- No space constraints or color figure charges

- Immediate publication on acceptance

- Inclusion in PubMed, CAS, Scopus and Google Scholar

- Research which is freely available for redistribution 